\title{
Non-spatial context-driven search
}

\author{
Sunghyun Kim ${ }^{1}$ - Melissa R. Beck ${ }^{1}$
}

Published online: 20 May 2020

(C) The Psychonomic Society, Inc. 2020

\begin{abstract}
Contexts that predict characteristics of search targets can guide attention by triggering attentional control settings for the characteristics. However, this context-driven search has most commonly been found in the spatial dimension. The present study explored the context-driven search when shape contexts predict the color of targets: non-spatial context-driven search. It has been demonstrated that context-driven search requires cognitive resources, and evidence of non-spatial context-driven search is found when there is an increase in cognitive resources for the shape/color associations. Thus, the scarcity of evidence for non-spatial context-driven search is potentially because the context-driven search requires more cognitive resources for shape/color associations than for spatial/spatial associations. In the current study, we violated a previously $100 \%$ consistent shape/color association with two mismatch trials to encourage allocation of cognitive resources to the shape/color association. Three experiments showed that the shape-predicted color cues captured attention more than the non-predicted color cues, indicating that shape contexts triggered attentional control settings for a color predicted by the contexts. Furthermore, the shape contexts guided attention to the predicted color only after the two mismatch trials, suggesting that expression of the non-spatial context-driven search may require cognitive resources more than the spatial context-driven search.
\end{abstract}

Keywords Attention · Visual search · Attention: Selective

\section{Introduction}

The visual sensory system receives a great deal of information, but attentional limitations severely restrict the amount of information processed beyond a sensory level. Attention is not only passively oriented by bottom-up saliency of objects, but also actively oriented by top-down attentional control settings of observers (Wolfe, 1994). Accordingly, it is important to understand how and when top-down attentional control settings are formed and when they are used (Awh, Belopolsky, \& Theeuwes, 2012). Top-down attentional control settings for target characteristics can be formed based on a learned context/characteristic association, but this has most commonly been reported for spatial characteristics (Brockmole, Castelhano, \& Henderson, 2006; Chun, 2000; Chun \& Jiang, 1998, 2003; Jiang \& Wagner, 2004; Olson \& Chun, 2002; Vaidya, Huger, Howard, \& Howard, 2007). The present study explores attentional control settings for non-spatial characteristics formed through incidental associative learning. In

Melissa R. Beck mbeck@1su.edu

1 Department of Psychology, Louisiana State University, 239 Audubon Hall, Baton Rouge, LA 70803, USA particular, when shape contexts predict the color of targets, can the shape contexts trigger an attentional control setting for the predicted color?

Visual scenes often contain structured properties of repeated patterns and regularities that may be used to guide attention. The structured properties can be extracted and learned without intention through an incidental learning process (Baker, Olson, \& Behrmann, 2004; Beck, Angelone, Levin, Peterson, \& Varakin, 2008; Beck, Goldstein, van Lamsweerde, \& Ericson, 2018; Fiser \& Aslin, 2005; Kirkham, Slemmer, \& Johnson, 2002; Leber, Gwinn, Hong, \& O'Toole, 2016; Otsuka, Nishiyama, Nakahara, \& Kawaguchi, 2013; Turk-Browne, Jungé, \& Scholl, 2005). Accordingly, while attentional control settings are largely considered to be explicitly formed (Folk, \& Remington, 1998; Wolfe, 1994), they can also be established for contexts through incidental associative learning: context-driven search (Brockmole, Castelhano, \& Henderson, 2006; Chun, 2000; Chun \& Jiang, 1998, 1999; Endo \& Takeda, 2004; Jiang \& Chun, 2001; Torralba, Oliva, Castelhano, \& Henderson, 2006). Context-driven search occurs when a learned association between a context and a target characteristic allows the context to trigger an attentional control setting for the target characteristic. 
Contextual cueing (Brockmole et al., 2006; Chun, 2000; Chun \& Jiang, 1998, 2003; Jiang \& Wagner, 2004; Olson \& Chun, 2002; Vaidya, Huger, Howard, \& Howard, 2007) is a specific example of spatial context-driven search in which the configuration of distractors predicts the location of a target. A typical methodology (e.g., Chun \& Jiang, 1998) of the spatial context-driven search is that configurations of distractors are occasionally repeated (repeated contexts) or not (novel contexts). The target location is predicted by the configuration of the distractors in the repeated contexts but not in the novel contexts, allowing faster detection of a search target for the repeated than novel contexts despite a lack of awareness of the associations between contexts and target locations. Incidental learning of the associations of the spatial characteristics leads to the repeated contexts triggering attentional control settings for target locations predicted by the contexts (Chun \& Jiang, 1998)

The present study investigated whether context-driven search occurs when shape contexts predict the target's color: non-spatial context-driven search. Evidence of shape contexts guiding attention to a color predicted by the contexts (e.g., Anderson, 2015; Gozli, Moskowitz, \& Pratt, 2014) is not as abundant as spatial context-driven search (Brockmole et al., 2006; Chun, 2000; Chun \& Jiang, 1998, 2003; Jiang \& Wagner, 2004; Jiang, Sigstad, \& Swallow, 2013; Olson \& Chun, 2002; Rosenbaum \& Jiang, 2013; see Goujon, Didierjean, \& Thorpe, 2015, for review). This is somewhat surprising given the ability to form attentional control settings for non-spatial features such as color and shape (Lamy \& Kristjánsson, 2013; Maunsell \& Treue, 2006). The imbalance in the amount of evidence supporting spatial and non-spatial context-driven search may be because non-spatial contextdriven search may require more cognitive resources than spatial context-driven search. Although context-driven search requires cognitive resources (Jiang \& Leung, 2005; Manginelli, Langer, Klose, \& Pollmann, 2013), non-spatial context-driven search appears to only be found in situations that encourage participants to devote additional cognitive resources to processing the shape/color associations (e.g., Anderson, 2015; Gozli et al., 2014).

The use of learned associations to guide attention requires cognitive resources (e.g., attention and working memory). Jiang and Leung (2005) demonstrated that although attention to contexts is not necessary for the associative learning in spatial context-driven search, attention is necessary for the contexts to trigger an attention bias (expression of the associative learning). Similarly, Manginelli et al. (2013) suggested that workingmemory resources are not required for the associative learning in the spatial context-driven search but are required for the expression of the previously learned associations. These findings suggest that although learning of the association may occur, spatial context-driven search may not occur if limited cognitive resources prevent processing of the contexts.
In order to use a learned shape/color association to guide attention during search toward the color predicted by the shape context, the need for cognitive resources might be greater than what has been shown for spatial context-driven search. Previous studies showing shape contexts triggering attentional control settings for color predicted by the contexts included methodological factors that could have increased cognitive resources for the shape/color association (Anderson, 2015; Gozli et al., 2014). In Anderson (2015), the associations between the shape contexts (background images) and color involved reward learning. Reward facilitates associative learning by prioritizing the reward-associated information (Hyman, Malenka, \& Nestler, 2006). Thus, the reward learning would increase cognitive resources for the shape/color associations (Anderson, 2015). In Gozli et al. (2014), the shape contexts did not guide attention to the shape-predicted color when the predictive shape (e.g., 'S') and predicted color (e.g., red) features were presented separately in different objects. However, they guided attention when both the shape and color features were presented in a single object (e.g., red "S"). The conjunctive presentation could have increased cognitive resources allocated to the shape/color association because the shape context ("S") was the target. That is, the shape context would receive attention regardless of the conjunctive presentation or separate presentation because the shape was the target. However, the color would receive less attention in the separate presentation than the conjunctive presentation because the color (non-target) would have attentional benefit when the color was presented with the shape (target) in a single object compared to when it was presented separately (Gozli et al., 2014). This is in line with research showing that attention to any one property of an object can facilitate attention to other properties of the object (Kahneman, Treisman, \& Gibbs, 1992). In the spatial context-driven search, however, such boosts for cognitive resources were not necessary (Brockmole et al., 2006; Chun, 2000; Chun \& Jiang, 1998, 2003; Jiang \& Wagner, 2004; Jiang, Sigstad, \& Swallow, 2013; Olson \& Chun, 2002; Rosenbaum \& Jiang, 2013). This discrepancy indicates that the non-spatial context-driven search may need more cognitive resources than the spatial context-driven search. In other words, to observe a shape context triggering an attentional control setting for a color associated with the shape, a factor that increases cognitive resources for the shape/color associations may be necessary.

The current study investigated whether a shape context can trigger attention for a color predicted by the context. Considering that a boost in cognitive resources may be needed to observe non-spatial context-driven search, we inserted two mismatch trials as a facilitator factor that increases cognitive resources to the shape/color association. The mismatch trials violated a previously consistent shape/color association. For example, square contexts predicted red targets, and circle contexts predicted green targets on all trials (match trials) except 
for the two mismatch trials. In the two mismatch trials, the associations were different (e.g., square/green, circle/red) from the match trials. The sudden conflict with the previous associations should increase cognitive resources for the shape/ color association by inducing the exploratory attention processes (Easdale, Le Pelley, \& Beesley, 2019; Hall \& Pearce, 1982; Pearce \& Bouton, 2001; Pearce \& Hall, 1980; Swan \& Pearce, 1988) and conflict-driven cognitive control (Botvinick, Cohen, \& Carter, 2004; Egner, 2007; Mayr, Awh, \& Laurey, 2003).

The mismatch trials would increase cognitive resources for the shape/color association by inducing exploratory attention. In associative learning, a sudden violation of the previously learned association between the predictor and outcome can increase attention to the predictor (Easdale, Le Pelley, \& Beesley, 2019; Griffiths, Johnson, \& Mitchell, 2011; Hall \& Pearce, 1982; Pearce \& Bouton, 2001; Pearce \& Hall, 1980; Swan \& Pearce, 1988; Wills, Lavric, Croft, \& Hodgson, 2007; Wilson, Boumphrey, \& Pearce, 1992). Specifically, the violation brings about uncertainty about the previous association. Then, an effort is made to resolve the uncertainty through exploratory attention. Exploratory attention increases attention to the uncertain stimuli. Therefore, the mismatch trials, which violate the previous associations, would induce exploratory attention to resolve the shape/color association, and so cognitive resources allocated to the shape/color association should increase.

The mismatch trials could also lead to an increase in conflict-driven cognitive control, which would increase the cognitive resources allocated to the shape/color association. Detection of cognitive conflict induces an up-regulation in cognitive control (Egner, 2007). The increased cognitive control leads to an increase in cognitive resources for taskrelevant information. This conflict-driven cognitive control effect occurs in various tasks (e.g., Simon, Stroop, Flanker tasks) and stimuli (Egner, 2007; Egner \& Hirsch, 2005). In the Stroop task, for example, the Stroop effect decreases after an incongruent trial where conflict occurs between a word meaning and the color of the word compared to after a congruent trial where no conflict occurs. This decrease in the Stroop effect is attributed to conflict from the incongruent trial leading to an increase in cognitive control. If the shape/color association is learned in the current study, the mismatch trials could induce conflict by violating the learned association. Therefore, we predicted that the mismatch trials would enhance cognitive control, and so cognitive resources for the shape/color association would increase.

\section{The present study}

The purpose of the present study was to investigate whether contexts predicting the color of targets can guide attention by triggering an attentional control setting for the target color (non-spatial context-driven search). To examine this non- spatial context-driven search, we developed the contextual pre-cueing paradigm. This paradigm is based on the precueing paradigm of Cosman and Vecera (2014) in which an attentional control setting for a particular color is formed through incidental learning of statistical information. Specifically, in Cosman and Vecera's (2014) pre-cueing paradigm, a search target was defined as the identity of a letter (e.g. "H" or "B"). Two letters (a target letter, a non-target letter) were presented in either red or green font, one on each side of a central fixation marker, and participants reported the identity of the target as quickly as possible. During the learning phase, unbeknownst to participants, one target color was more probable than the other (e.g., $80 \%$ red, $20 \%$ green). During the test phase, target color was no longer biased. Results from the test phase showed that cues presented prior to the letter stimuli captured attention more when the cues were the probable target color (red cues) than when they were the less probable target color (green cues), suggesting that participants incidentally formed an attentional control setting for the probable target color (Cosman \& Vecera, 2014).

The current study expanded on this finding by creating a contextual pre-cueing paradigm in which one of two contexts (square placeholders and circle placeholders) was randomly presented from trial to trial. Participants reported if a $\mathrm{Z}$ or an $\mathrm{N}$ was presented. The targets $(\mathrm{Z}$ or $\mathrm{N})$ appeared in one of two target colors (red or green) that were equally probable. The context (square or circle) predicted the color of the search target, allowing us to test if an attentional control setting for a specific color can be triggered by an associated context (i.e., "context A = red target" and "context B = green target" associations induce searching for red under context $\mathrm{A}$ and green under context B). If non-spatial context-driven search occurs, cues that have the same color as the context-predicted target color (red cues under context A and green cues under context B) would capture attention more than cues that do not have the same color (green cues under context A and red cues under context B). Accordingly, the cueing effect (faster responses for targets appearing at a cued than an un-cued location) would be larger for the context-predicted color cues than for the non-predicted color cues.

\section{Experiment 1}

Experiment 1 investigated whether an attentional control setting for a color can be flexibly adopted depending on a given context as the result of incidental learning of the associations between contexts and color of targets. In the contextual precueing paradigm, participants were asked to find letters $\mathrm{Z}$ and N, only one of which was presented on each trial (see Fig. 1). The target letter was colored in either red or green and the distracter letter was colored in the other color. Critically, each target color was paired with a particular context. For example, 

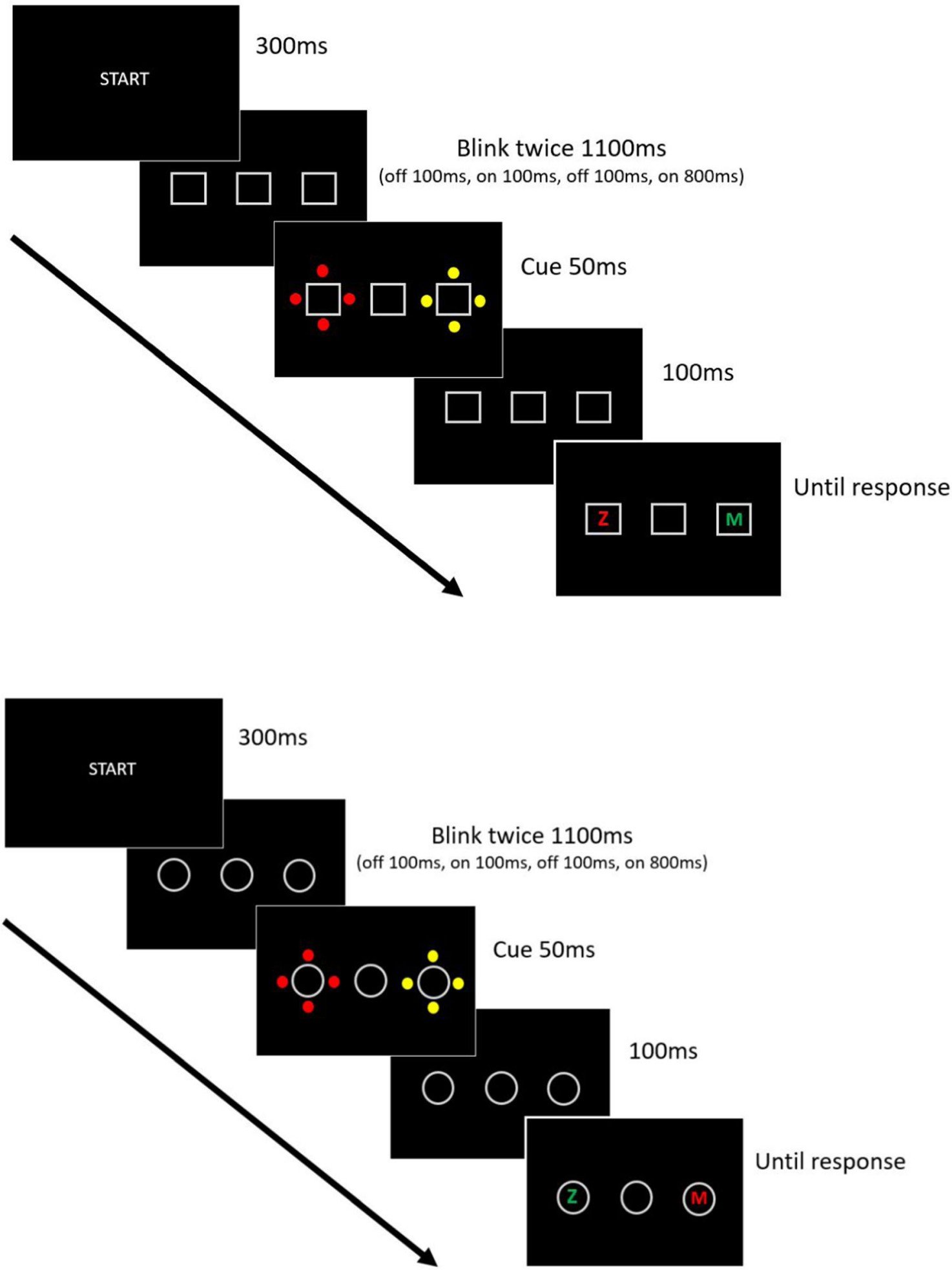

Fig. 1 Examples of the square (top) and circle (bottom) contexts. A cue color was either red or green, and the other location was always yellow. In the examples, when the context was square (circle), the color of the targets $(\mathrm{Z}$ or $\mathrm{N})$ was red (green). Therefore, a red cue was the predicted color cue in the square context, but the non-predicted color cue in the circle context. In addition, a valid (invalid) cue was when locations between a cue and target were the same (different). Therefore, in the examples, both red cues in the square and circle contexts are valid cues the search target was red when the shape of the fixation marker and placeholders was square, and it was green when the shape was a circle. Participants were not informed of the shape/color association. 
If the contexts (shape of the fixation marker and placeholders) trigger attentional control settings for the color predicted by the contexts, predicted color cues (i.e., cues with the same color as the target color predicted by a given context) would better capture attention than the non-predicted color cues (i.e., cues with a different color from the target color predicted by a given context). For example, when the square context was paired with red targets, red cues were predicted color cues with the square context but non-predicted color cues with the circle context. Therefore, when there is an attentional set based on the context-target color association, predicted color cues should capture attention more than nonpredicted color cues, leading to a cueing effect (shorter response time when cues validly cue the target location than when the cues are invalid) that is larger for the predicted than non-predicted color cue. Accordingly, a larger cueing effect for predicted color than non-predicted color cues is an indication of a non-spatial context-driven search effect.

We predicted that non-spatial context-driven search may not occur without a violation in the association that could increase cognitive resources allocated to the shape/color association. Following learning trials in which a given context was always paired with the same target color, two mismatch trials were added in which the context-color association was violated. Mismatch trials should increase exploratory attention (Easdale, Le Pelley, \& Beesley, 2019; Griffiths, Johnson, \& Mitchell, 2011; Hall \& Pearce, 1982; Pearce \& Bouton, 2001; Pearce \& Hall, 1980; Swan \& Pearce, 1988; Wills, Lavric, Croft, \& Hodgson, 2007; Wilson, Boumphrey, \& Pearce, 1992) and cognitive control (Egner, 2007) and, therefore, may provide the cognitive resources necessary for the use of the learned shape-color associations to guide attention. Furthermore, we predicted that the effect triggered by the mismatch trials would be short lived. The exploratory attention and conflict-driven cognitive control are not long lasting (Kaye \& Pearce, 1984; Rey-Mermet \& Meier, 2017). Thus, the two mismatch trials would facilitate the expression of the non-spatial context-driven search by temporarily increasing cognitive resources to the shape/color association. We predicted that epochs of trials just before the mismatch trials would not show a larger cueing effect for the predicted than the nonpredicted color cue, but epochs of trials just after the mismatch trials would show the effect.

\section{Method}

Participants Twenty-four undergraduate students participated for course credit $(21$ females, average age $=18.25$ years, $\mathrm{SD}=$ .61). All had normal or corrected-to-normal vision. Sample size was on the basis of the Gozli et al. (2014) study where cue validity differences between cue colors, which is the indication of a non-spatial context-driven search effect, had an effect size of $\eta_{p}^{2}=.33$. G power analysis (a power of 0.80 , an alpha of 0.05 , effect size of $\eta_{p}^{2}=.33$ ) indicated the minimum sample size of 19 .

Apparatus Stimuli were presented on a monitor (20-in.) of a MacOS computer. The distance between the participants and monitor was approximately $60 \mathrm{~cm}$ but was not constrained. Experiments were programmed using MATLAB and Psychophysics Toolbox software.

Stimuli, procedure, and design All stimuli were presented on a black background. For the square context trial (see Fig. 1), the fixation marker $\left(1.3^{\circ}\right.$ each side of a square) and placeholders $\left(1.3^{\circ}\right.$ each side of a square) were squares. For the circle context trial (see Fig. 1), the fixation marker (1.4 ${ }^{\circ}$ diameter) and placeholders $\left(1.4^{\circ}\right.$ diameter) were circles. At the beginning of each trial, a text message of "START" was presented for $300 \mathrm{~ms}$ at the center of a black screen. Then, after a blank, all black, display for $100 \mathrm{~ms}$, the fixation marker and placeholders (two circles or two squares on either side of the fixation marker) were presented for $100 \mathrm{~ms}$, followed by another blank display of $100 \mathrm{~ms}$. The fixation marker and placeholders then reappeared after $800 \mathrm{~ms}$. The cue display was presented for $50 \mathrm{~ms}$, where one of the placeholders was cued with four red (RGB: 255, 0, 0) or green (RGB: $0,255,0)$ small dots around the cued placeholder, and the other placeholder always had four yellow dots (RGB: 255, 255, 0). After the cue disappeared, the fixation marker and empty placeholders remained on-screen for $100 \mathrm{~ms}$. Then, one of the search targets $(\mathrm{Z}$ and $\mathrm{N})$ was presented in one of the placeholders and one of the distractors $(\mathrm{M}, \mathrm{V}$, and $\mathrm{X})$ was presented in the other placeholder until a response was made. The participants were asked to find and identify the search target by pressing the corresponding key ( $\mathrm{Z}$ or $\mathrm{N})$ as quickly and accurately as possible. Feedback was provided directly after a response was made for slow or incorrect responses. If a correct, but slow, response was made (response time greater than 1,000 ms), a message of "too slow" was presented in the center of the screen for $700 \mathrm{~ms}$. If an incorrect response was made regardless of response time, a message of "wrong" was presented in the center of the screen for 700 $\mathrm{ms}$. If a correct response was made before $1,000 \mathrm{~ms}$, no feedback was presented, and the next trial began. Participants were explicitly informed that the shape of the fixation marker and placeholders would change between square and circle. However, they were not informed of any possible association between the shape and the target color.

The experiment consisted of a practice session (20 trials; participants had opportunities to ask questions during and right after practice), an experiment session (800 match trials, ${ }^{1}$

\footnotetext{
${ }^{1}$ The first 12 of 812 trials were excluded because every 16 trials contained an equal number of trials for each condition and this was applied from the beginning of the practice session, which had 20 trials. The exclusion allowed the analysis of the 800 trials containing an equal number of trials for each condition.
} 
two mismatch trials, and 128 match trials in sequence), and a post-test questionnaire. A self-paced break was given after the 800th trial of the experiment session. Two mismatch trials were inserted on the 801 st and 802 nd trials. In the match trials, for half of the participants, the color of the search target was always red for the square context, and the color of the search target was always green for the circle context. For the other half of participants, this association was reversed. The distractor was always the other color (i.e., if the target was red, the distractor was green).

For each context, the cue color was equally likely to be either the context-predicted target color (predicted color cue) or the context-predicted distractor color (non-predicted color cue). Therefore, within each context, the color of the cue was equally often either red or green. When the cue color was the same as the target color, which was predicted by a context, the cue was the predicted color cue. When the cue color was not the same as the predicted target color but was the same as the predicted distractor letter color, the cue was the non-predicted color cue. Note that although contexts predict the color of both the target and distractor letters (e.g., target and distractor colors are always red and green, respectively, in the square context), the predictive relationship (context-predicted color, context-non-predicted color) was based on the association between the contexts and target color. For example, in the square-red target association, the predicted color cue was red (target color in the square context) and the nonpredicted color cue was green (distractor color in the square context).

Two critical factors were manipulated across trials: cue validity (valid or invalid cue), and cue color (predicted or non-predicted color cue). Valid cues were presented at the same location as the target. Invalid cues were presented at the other location. Within every 16 consecutive trials in the practice and experiment sessions, contexts (square or circle), cue color (red or green), cue location (left or right), and target location (left or right) were fully crossed, so that every 16 trials included four valid predicted color cue trials, four valid non-predicted color cue trials, four invalid predicted color cue trials, and four invalid non-predicted color cue trials. A target type $(\mathrm{Z}$ or $\mathrm{N})$ and distractor type $(\mathrm{M}, \mathrm{V}$, and $\mathrm{X})$ were randomly selected on each trial.

The two mismatch trials had reversed context-color associations (Fig. 2). For example, if original associations were square context-red target and circle context-green target, reversed associations were square context-green target and circle context-red target. Immediately after the experiment, a post-test awareness question was given to check awareness of the association between contexts and color of targets. This was an open-ended question in which participants were asked to describe any patterns, in terms of the shape of the fixation and placeholders that they had noticed.

\section{Results}

Analyses of variance (ANOVAs) were conducted on the mean of response times (RTs) with cue validity (valid or invalid cue) and cue color (predicted or non-predicted color cue) as withinsubjects variables for each epoch. Each epoch had 48 trials (12 valid predicted color cue, 12 invalid predicted color cue, 12 valid non-predicted color cue, and 12 invalid non-predicted color cue). The 705th -752 nd trials, 753rd -800 th trials, 803rd -850 th trials, and 851 st -898 th trials belonged to epochs 1, 2, 3, and 4, respectively. The two mismatch trials were presented only on the 801st and 802 nd trials. The two mismatch trials were excluded from the analysis (Pearce \& Hall, 1980). The analyses were restricted to these four epochs to check for a cueing effect before and after the mismatch trials.

It should be noted that the epoch size (48 trials) was used so that there was an equal number of each trial type in each epoch and was determined based on initial visual inspection of the results of Experiment 1. The post-decision of the epoch size was used because although the exploratory attention and conflict-driven cognitive control are not long lasting (Kaye \& Pearce, 1984; Rey-Mermet \& Meier, 2017), the length of them in the methodology of the present study was unknown. The epoch size of 48 trials was maintained for the data analysis in Experiments 2 and 3 to confirm that the epoch size could be used to replicate the effect.

RT was measured from the onset of a target until a response was made. Overall, $15.49 \%$ of trials were excluded from analysis: RTs shorter than $125 \mathrm{~ms}(0.07 \%)$, RTs longer than $1,000 \mathrm{~ms}(4.94 \%)$, and incorrect responses $(10.47 \%)$. The $1,000-\mathrm{ms}$ exclusion was based on criteria used in Cosman and Vecera (2014).

Response time To examine if the non-spatial context-driven search effect occurred only immediately after the mismatch trials, ANOVAs with cue color and validity were conducted for epochs $1-4$ where epochs 1 and 2 occurred just before the mismatch trials and epochs 3 and 4 occurred just after the mismatch trials. In epoch 1 , the main effects of cue color and validity were not significant, $F(1,23)=1.17, p=.29, \eta_{p}^{2}$ $=.05$, and $F(1,23)=2.32, p=.14, \eta_{p}^{2}=.09$, respectively. The interaction between cue color and validity was also not significant, $F(1,23)=2.00, p=.17, \eta_{p}^{2}=.08$. In epoch 2 , the main effects of cue color and validity were not significant, $F(1,23)$ $=1.94, p=.18, \eta_{p}^{2}=.08$, and $F(1,23)<.01, p=.96, \eta_{p}^{2}<.01$, respectively. The interaction between cue color and validity was also not significant, $F(1,23)=.24, p=.63, \eta_{p}^{2}=.01$. In epoch 3 , the main effects of cue color and validity were not significant, $F(1,23)=.18, p=.68, \eta_{p}^{2}=.01$, and $F(1,23)=$ $1.00, p=.76, \eta_{p}^{2}<.01$, respectively. However, the interaction 

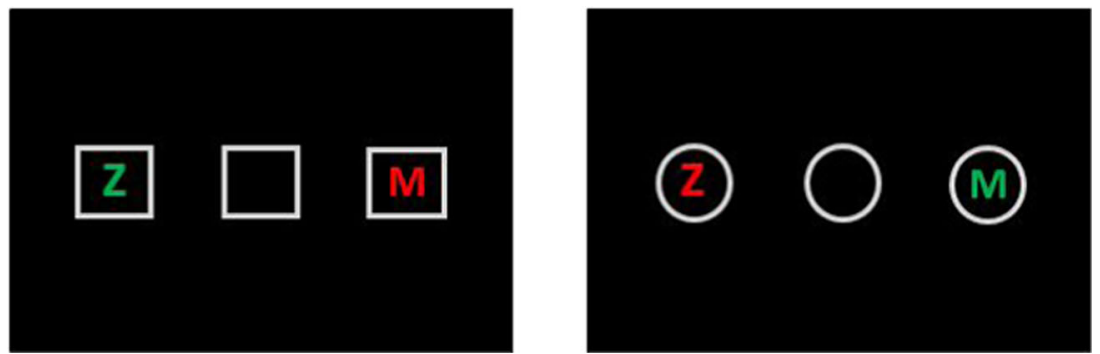

Fig. 2 Examples of the reversed color mismatch trials used in Experiment 1. These examples were when the original associations (match trials) were square-red and circle-green

between cue color and validity was significant, $F(1,23)=$ $5.38, p=.03, \eta_{p}^{2}=.19$, due to a larger validity effect for the predicted than the non-predicted color cue (the non-spatial context-driven search effect). Post hoc tests further revealed that the predicted color cue produced a significant validity effect [19 ms; $t(23)=2.31, p=.03$ ], while the non-predicted color cue did not $[-14 \mathrm{~ms} ; t(23)=-1.16, p=.26]$. In epoch 4 , the main effects of cue color and validity were not significant, $F(1,23)=.80, p=.38, \eta_{p}^{2}=.03$, and $F(1,23)<.01, p=.98, \eta_{p}^{2}$ $<.01$, respectively. The interaction between cue color and validity was not significant, $F(1,23)=.72, p=.40, \eta_{p}^{2}=.03$. The cue validity effect (invalid RTs - valid RTs) for each type of cue and each epoch is reported in Fig. 3 (see Table 1 for invalid and valid RTs).

The lack of an interaction between cue color and cue validity in epochs 1 and 2 suggests that the non-spatial contextdriven search effect did not occur even after hundreds of match trials. To further confirm this, new ANOVAs with cue color and cue validity as within-subject factors were conducted on epochs of 80 trials (1-80, 81-160, 161-240, 241320, 321-400, 401-480, 481-560, 561-640, 641-720, and 721-800 trials; see Fig. 3). However, no significant interactions between cue color and cue validity (no non-spatial context-driven search effect) were found in any of the epochs (all $p s>.19$; see Appendix Table 7 for a table of the ANOVA values).

Accuracy None of the interactions or main effects of cue color and validity were significant in any of the epochs. In epoch 1 , the main effects of cue color and validity were not significant, $F(1,23)=1.48, p=.23, \eta_{p}^{2}=.06$, and $F(1,23)=1.95, p=.17$, $\eta_{p}^{2}=.07$, respectively. The interaction between cue color and validity was not significant, $F(1,23)=.01, p=.90, \eta_{p}^{2}<.01$. In epoch 2 , the main effects of cue color and validity were not significant, $F(1,23)=.34, p=.56, \eta_{p}^{2}=.01$, and $F(1,23)=.15$, $p=.69, \eta_{p}^{2}<.01$, respectively. The interaction was not significant, $F(1,23)=3.24, p=.08, \eta_{p}^{2}=.12$. In epoch 3 , the main effects of cue color and validity were not significant, $F(1,23)$ $=.77, p=.38, \eta_{p}^{2}=.03$, and $F(1,23)=.14, p=.71, \eta_{p}^{2}<.01$, respectively. The interaction was not significant, $F(1,23)=$
$.33, p=.57, \eta_{p}^{2}=.01$. Furthermore, Bayes factor $\left(\mathrm{BF}_{01}\right)=3.2$ indicated that the null was 3.2 times more likely than the interaction, supporting the premise that the non-spatial context-driven search effect in RT was not due to speed-accuracy tradeoff. In epoch 4 , the main effects of cue color and validity were not significant, $F(1,23)=.59, p=.45, \eta_{p}^{2}=.02$, and $F(1$, $23)=2.04, p=.16, \eta_{p}^{2}=.08$, respectively. The interaction between cue color and validity was also not significant, $F(1$, 23) $=1.14, p=.29, \eta_{p}^{2}=.05$ (see Table 2$)$.

Awareness question No participant reported noticing the association between the context and color of the target.

\section{Discussion}

The current experiment demonstrated the non-spatial contextdriven search only after the mismatch trials. Specifically, after the mismatch trials, the shape contexts triggered attentional control setting for colors predicted by the shape contexts. Accordingly, the shape-predicted color cues captured attention more than the non-predicted color cues, showing a larger cueing effect for the predicted than the non-predicted color cues. Additionally, consistent with the previous findings (Anderson, 2015; Gozli et al., 2014), the shape context guiding attention to the shape-associated color was found under circumstances (mismatch trials) likely to increase cognitive resources for the shape/color association (epoch 3 ). Although the effect after the mismatch trials demonstrates that the association was learned before the mismatch trials, the association was not used to guide attention until the mismatch trials disrupted the association.

\section{Experiment 2}

Experiment 2 aimed to replicate the results of Experiment 1. It was important to see if the use of the epoch size of 48 trials would be effective in a new experiment given that the epoch size was determined based on visual inspection of the pattern of results in Experiment 1. The exploratory attention and conflict-driven cognitive control are known to not be long 


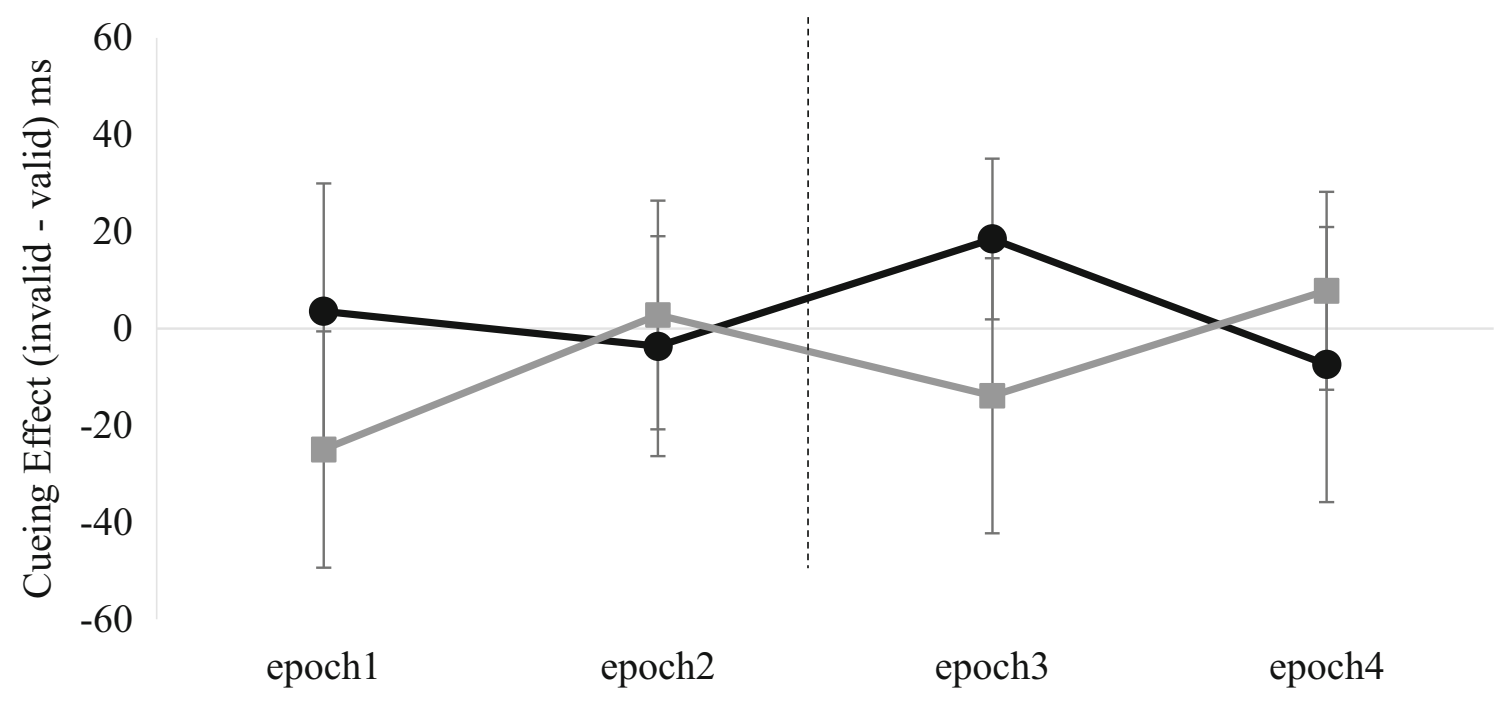

$\rightarrow-$ Predicted Color Cue $\quad-$-Non-Predictied Color Cue

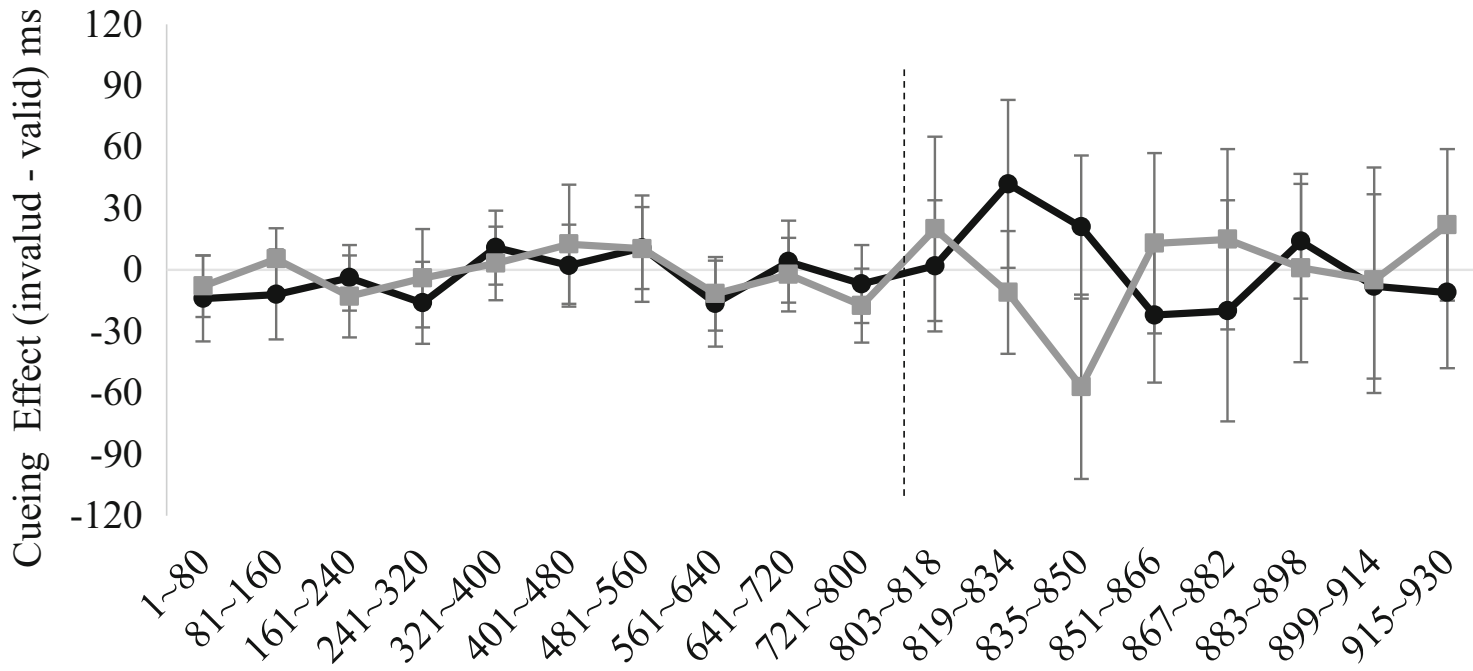

Trials

Fig. 3 Results of Experiment 1. In the top graph, each of epochs 1-4 includes 48 trials and across the following range of trials: 705-752, 753$800,803-850$, and $851-898$, respectively. The bottom graph is the data in bins of 80 trials until the mismatch trials and then bins of 16 trials after the

Table 1 Mean response times and standard error of the mean (in milliseconds) as a function of epoch, cue color, and cue validity in Experiment 1

\begin{tabular}{llllll}
\hline & \multicolumn{2}{l}{ Predicted color } & & \multicolumn{2}{l}{ Non-predicted color } \\
\cline { 2 - 3 } \cline { 6 - 6 } & Valid & Invalid & & Valid & Invalid \\
\hline Epoch 1 & $594(11)$ & $598(12)$ & & $600(12)$ & $575(9)$ \\
Epoch 2 & $595(14)$ & $591(13)$ & & $580(10)$ & $582(15)$ \\
Epoch 3 & $561(12)$ & $580(12)$ & & $573(10)$ & $559(12)$ \\
Epoch 4 & $589(11)$ & $582(9)$ & & $575(9)$ & $583(7)$ \\
\hline
\end{tabular}

mismatch trials. The mismatch trials occurred between epochs 2 and 3, on trials 801 and 802 (see dashed black vertical line). Error bars represent $95 \%$ confidence intervals

Table 2 Mean of accuracy and standard error of the mean (in percentage) as a function of epoch, cue color, and cue validity in Experiment 1

\begin{tabular}{llllll}
\hline & \multicolumn{2}{l}{ Predicted color } & & \multicolumn{2}{l}{ Non-predicted color } \\
\cline { 2 - 3 } \cline { 6 - 6 } \cline { 6 - 6 } & Valid & Invalid & & Valid & Invalid \\
\hline Epoch 1 & $90.3(2.4)$ & $88.5(2.4)$ & & $92(1.8)$ & $90.6(2.4)$ \\
Epoch 2 & $84.7(3.0)$ & $89.6(2.5)$ & & $89.9(2.1)$ & $86.5(2.8)$ \\
Epoch 3 & $91(2.2)$ & $90.6(1.7)$ & & $88.9(1.9)$ & $90.6(2.0)$ \\
Epoch 4 & $86.5(2.5)$ & $90.6(1.8)$ & & $90.3(2.3)$ & $88.5(2.2)$ \\
\hline
\end{tabular}


lasting (Kaye \& Pearce, 1984; Rey-Mermet \& Meier, 2017), and Experiment 1 suggests that the effect is gone in as short as 48 trials. The purpose of Experiment 2 was to test if these findings of Experiment 1 would generalize and be replicated when using a different type of mismatch trial. Another typical way of violating regularities in the incidental associative learning is omitting an expected event (e.g., Den Ouden, Friston, Daw, McIntosh, \& Stephan, 2008). Experiment 1 swapped the association on the mismatch trials; therefore, although the association was broken, the expected color (red or green) was seen in the search display. In contrast, on the two mismatch trials in Experiment 2, the target and distractor letters were gray (see Fig. 4). Like the mismatch trials of Experiment 1, the new type of mismatch trials also conflicted with the match trials, and therefore would likely increase cognitive resources for the shape/color association by inducing exploratory attention and conflict-driven cognitive control. Therefore, we predicted that the pattern of results as found in Experiment 1 would be replicated.

\section{Method}

Participants Twenty-four undergraduate students participated for course credit ( 17 females, average age $=19.33$ years, $S D=$ 2.81). All had normal or corrected-to-normal vision.

Apparatus, stimuli, procedure, and design Experiment 2 was the same as Experiment 1 except for the two mismatch trials where the color of the target and distractor letters was gray (Fig. 4).

\section{Results}

Overall, $13.29 \%$ of trials were excluded from analysis: Trials where RT was shorter than $125 \mathrm{~ms}(0.03 \%)$ or longer than $1,000 \mathrm{~ms}(5.20 \%)$ and trials where an incorrect response was made $(8.06 \%)$.

Response time In epoch 1, the main effects of cue color and validity were not significant, $F(1,23)=.60, p=.45, \eta_{p}^{2}=.03$, and $F(1,23)=3.12, p=.09, \eta_{p}^{2}=.12$, respectively. The

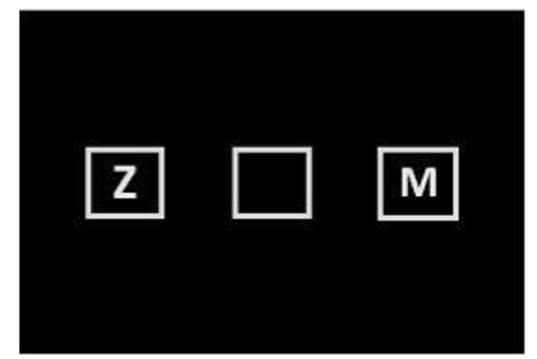

Fig. 4 Examples of gray mismatch trials used in Experiment 2 interaction between cue color and validity was also not significant, $F(1,23)<.01, p=.97, \eta_{p}^{2}<.01$. In epoch 2 , the main effects of cue color and validity were not significant, $F(1,23)$ $=.16, p=.70, \eta_{p}^{2}=.01$, and $F(1,23)=3.87, p=.06, \eta_{p}^{2}=.14$, respectively. The interaction between cue color and validity was also not significant, $F(1,23)<.01, p=.97, \eta_{p}^{2}<.01$. In epoch 3 , the main effects of cue color and validity were not significant, $F(1,23)=1.00, p=.33, \eta_{p}^{2}=.04$, and $F(1,23)$ $=.18, p=.67, \eta_{p}^{2}=.01$, respectively. However, the interaction between cue color and validity was significant, $F(1,23)=$ $6.86, p=.015, \eta_{p}^{2}=.23$, and was due to a larger validity effect for the predicted than non-predicted color cue (the non-spatial context-driven search effect). Post hoc tests further revealed that the predicted color cue produced a significant validity effect [20 ms; $t(23)=2.19, p=.04]$ while the non-predicted color cue did not [-15 ms; $t(23)=-1.63, p=.12]$. In epoch 4 , the main effects of cue color and validity were not significant, $F(1,23)=.04, p=.84, \eta_{p}^{2}<.01$, and $F(1,23)<.01, p=.97, \eta_{p}^{2}$ $<.01$, respectively. The interaction between cue color and validity was also not significant, $F(1,23)=.12, p=.73, \eta_{p}^{2}$ $<.01$. The cue validity effect (invalid RTs - valid RTs) for each type of cue and each epoch is reported in Fig. 5 (see Table 3 for invalid and valid RTs).

To further confirm the lack of non-spatial context-driven search effect before the mismatch trials, new ANOVAs (see Appendix Table 8) on cue color and cue validity were conducted for RT averaged across every 80 trials $(1-80,81-160$, 161-240, 241-320, 321-400, 401-480, 481-560, 561-640, 641-720, and 721-800 trials; see Fig. 5). The seventh interval (481-560 trials) of ten intervals showed a significant interaction between cue color and cue validity, $F(1,23)=6.03, p=$ $.02, \eta_{p}^{2}=.21$; however, the other nine intervals did not, indicating no reliable effect.

Accuracy None of the interactions or main effects of cue color and validity were significant in any of the epochs. In epoch 1 , the main effects of cue color and validity were not significant, $F(1,23)=.01, p=.92, \eta_{p}^{2}<.01$, and $F(1,23)=.10, p=.74, \eta_{p}^{2}$ $<.01$, respectively. The interaction between cue color and validity was also not significant, $F(1,23)=.01, p=.90, \eta_{p}^{2}$

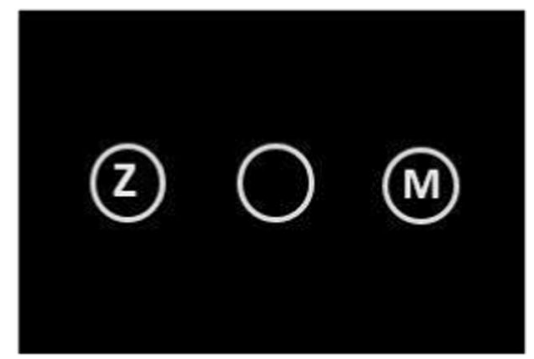




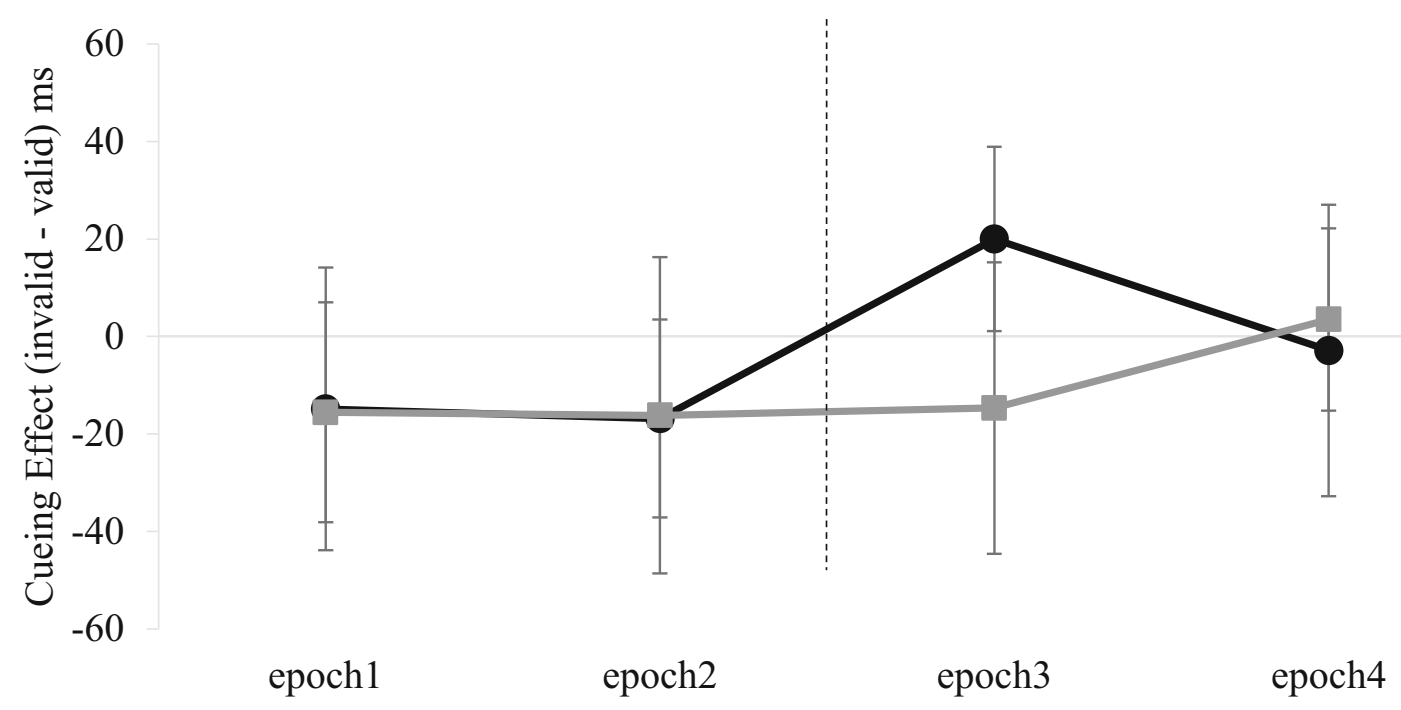

$\multimap$ Predicted Color Cue $\quad-$ Non-Predicted Color Cue

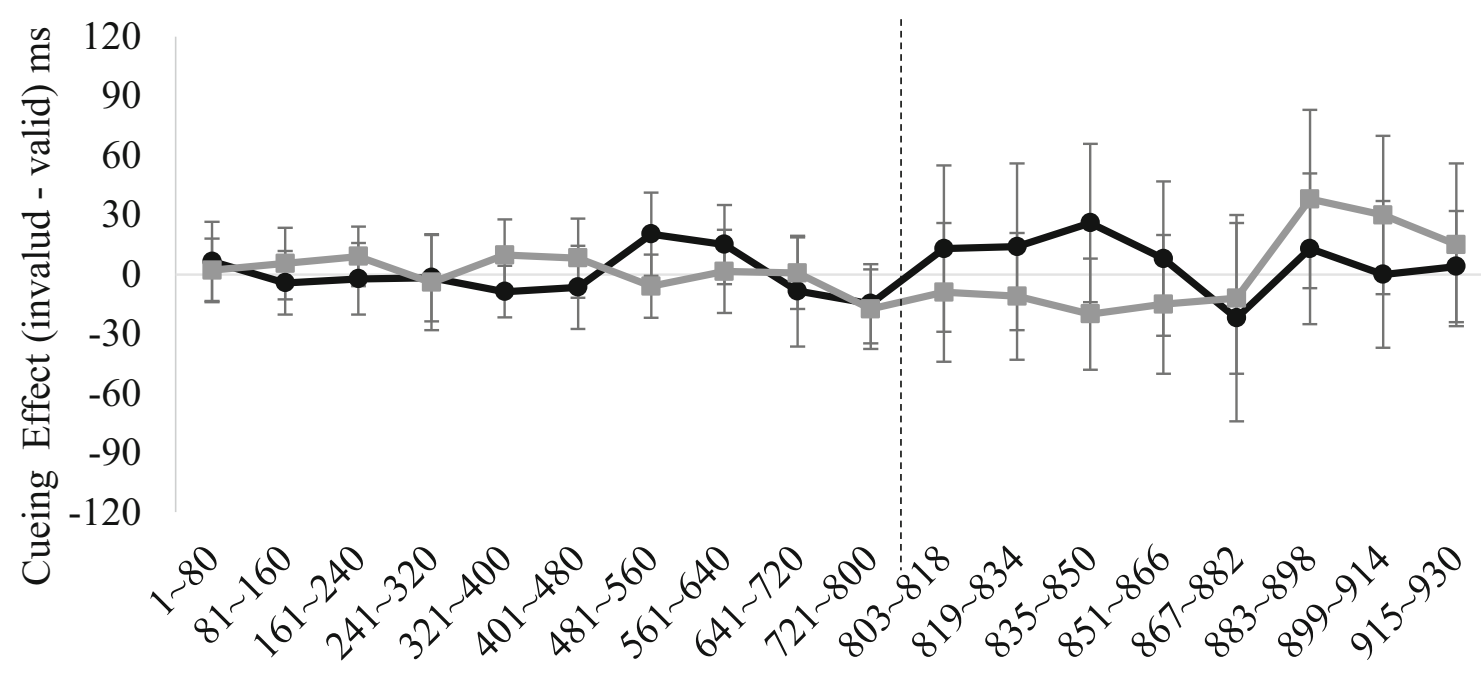

Trials

Fig. 5 Results of Experiment 2. In the top graph, each of epochs 1-4 includes 48 trials and across the following range of trials: 705-752, 753$800,803-850$, and $851-898$, respectively. The bottom graph is the data in bins of 80 trials until the mismatch trials and then bins of 16 trials after the

Table 3 Mean response times and standard error of the mean (in milliseconds) as a function of epoch, cue color, and cue validity in Experiment 2

\begin{tabular}{llllll}
\hline & \multicolumn{2}{l}{ Predicted color } & & \multicolumn{2}{l}{ Non-predicted color } \\
\cline { 2 - 3 } \cline { 6 - 6 } & Valid & Invalid & & Valid & Invalid \\
\hline Epoch 1 & $587(20)$ & $572(15)$ & & $580(13)$ & $564(14)$ \\
Epoch 2 & $577(15)$ & $560(16)$ & & $580(16)$ & $564(18)$ \\
Epoch 3 & $541(12)$ & $561(11)$ & & $552(12)$ & $537(12)$ \\
Epoch 4 & $554(14)$ & $551(13)$ & & $550(11)$ & $553(12)$ \\
\hline
\end{tabular}

mismatch trials. The mismatch trials occurred between epochs 2 and 3, on trials 801 and 802 (see dashed black vertical line). Error bars represent $95 \%$ confidence intervals

Table 4 Mean of accuracy and standard error of the mean (in percentage) as a function of epoch, cue color, and cue validity in Experiment 2

\begin{tabular}{llllll}
\hline & \multicolumn{2}{l}{ Predicted color } & & \multicolumn{2}{l}{ Non-predicted color } \\
\cline { 2 - 3 } \cline { 6 - 6 } \cline { 6 - 6 } & Valid & Invalid & & Valid & Invalid \\
\hline Epoch 1 & $91(2.3)$ & $91.3(1.7)$ & & $91(1.9)$ & $91.7(1.8)$ \\
Epoch 2 & $92.7(1.8)$ & $91.3(1.9)$ & & $88.5(2.1)$ & $93.8(1.7)$ \\
Epoch 3 & $92(1.8)$ & $91.3(2.3)$ & & $92(1.5)$ & $93.4(1.9)$ \\
Epoch 4 & $89.9(1.9)$ & $90.6(2.0)$ & & $91.3(1.8)$ & $92.4(1.4)$ \\
\hline
\end{tabular}


$<.01$. In epoch 2 , the main effects of cue color and validity were not significant, $F(1,23)=.30, p=.58, \eta_{p}^{2}=.01$, and $F(1$, 23) $=1.70, p=.20, \eta_{p}^{2}=.07$, respectively. The interaction was not significant, $F(1,23)=3.68, p=.07, \eta_{p}^{2}=.13$. In epoch 3 , the main effects of cue color and validity were not significant, $F(1,23)=.50, p=.48, \eta_{p}^{2}=.02$, and $F(1,23)=.03, p=.85, \eta_{p}^{2}$ $<.01$, respectively. The interaction was not significant, $F(1$, $23)=.65, p=.42, \eta_{p}^{2}=.03$. Furthermore, Bayes factor $\left(\mathrm{BF}_{01}\right)$ $=3.05$ suggested that the null was 3.05 times more likely than the interaction, supporting the non-spatial context-driven search effect in RT was not due to speed-accuracy tradeoff. In epoch 4, the main effects of cue color and validity were not significant, $F(1,23)=.81, p=.37, \eta_{p}^{2}=.03$, and $F(1,23)=.80$, $p=.38, \eta_{p}^{2}=.03$, respectively. The interaction was not significant, $F(1,23)=.02, p=.88, \eta_{p}^{2}<.01$, (see Table 4$)$.

Awareness question No participant reported noticing the association between the context and color of the target.

\section{Discussion}

In Experiment 2 we used a different type of mismatch trial from Experiment 1 but the same epoch size as Experiment 1. Therefore, Experiment 2 generalized and replicated the findings of Experiment 1: the non-spatial context-driven effect occurred only immediately after the mismatch trials (epoch 3). Together, Experiments 1 and 2 demonstrate that contexts can trigger attentional control settings for a color predicted by a given context. In addition, like the previous studies (Anderson, 2015; Gozli et al., 2014), expression of the non-spatial context-driven search needed additional cognitive resources. Specifically, the addition of the mismatch trials increases allocation of cognitive resources (i.e., attention and working memory), temporarily allowing the use of the learned association to guide attention. Without this increase in resources, even though the association has been present for hundreds of trials, the association is not used to guide attention.

\section{Experiment 3}

In Experiments 1 and 2, a break was given just before the mismatch trials. Experiment 3 verified if the non-spatial context-driven search was influenced by the break rather than the mismatch trials. Thus, Experiment 3 was identical to Experiment 2 except for the absence of the break. Furthermore, the sample size for Experiments 1 and 2 was based on previous research, but the effect found in the current research is smaller than those found in previous research.
Therefore, we used the effect size from Experiment 2 to estimate the sample size needed in Experiment 3. In addition, in Experiments 1 and 2, we were operating with an apriori hypothesis that the effect would be found only in epoch 3 . However, a more conservative approach is needed to increase confidence in the validity of this effect. Therefore, in Experiment 3 we used a Bonferroni correction (alpha $=$ 0.0125 ) for multiple analyses across four epochs.

\section{Method}

Participants Forty-four undergraduate students participated for course credit (33 females, average age $=18.9$ years, SD $=1.45)$. The sample size was based on the effect size $\left(\eta_{p}^{2}=\right.$ .23 ) of the cue-validity difference between colors in epoch 3 of Experiment 2. G power (a power of 0.80, an alpha of 0.0125 adjusted by Bonferroni correction, the effect size of $\eta_{p}^{2}=.23$ ) indicated the minimum sample size, 42. All had normal or corrected-to-normal vision.

Apparatus, stimuli, procedure, and design Experiment 3 is identical to Experiment 2 except that the break before the mismatch trials was removed.

\section{Results}

Overall, $10.39 \%$ of trials were excluded from analysis: trials where RT was shorter than $125 \mathrm{~ms}(0.08 \%)$ or longer than $1,000 \mathrm{~ms}(3.39 \%)$ and trials where an incorrect response was made $(6.92 \%)$.

Response time In epoch 1, the main effects of cue color and validity and the interaction were not significant, $F(1$, $43)=.07, p=.80, \eta_{p}^{2}<.01, F(1,43)=.11, p=.74$, $\eta_{p}^{2}<.01$, and $F(1,43)=.54, p=.46, \eta_{p}^{2}=.01$, respectively. In epoch 2 , the main effects of cue color and validity and the interaction were not significant, $F(1$, $43)=.01, p=.92, \eta_{p}^{2}<.01, F(1,43)=1.16, p=.29$, $\eta_{p}^{2}=.03$, and $F(1,43)=1.04, p=.31, \eta_{p}^{2}=.02$, respectively. In epoch 3 , the main effects of cue color and validity were not significant, $F(1,43)=.25, p=.62$, $\eta_{p}^{2}<.01$, and $F(1,43)=.09, p=.75, \eta_{p}^{2}<.01$, respectively. Critically, the interaction between cue color and validity was significant, $F(1,43)=8.45, p=.006, \eta_{p}^{2}=$ .16 , indicating the non-spatial context-driven search effect. Post hoc tests further revealed that the predicted color cue produced a marginally significant validity effect $[11 \mathrm{~ms} ; t(43)=1.64, p=.10]$ and significant for the non-predicted color cue $[-14 \mathrm{~ms} ; t(43)=-2.09, p=$ .04]. As in Experiments 1 and 2, the interaction (nonspatial context-driven search effect) of epoch 3 appears 
to be the result of the trend of both the validity effect of the predicted color cue and the reversed validity effect of the non-predicted cue. This appears to be due to the contexts predicting both target color, which was the same color as the predicted color cue, and distractor color, which was the same color as the non-predicted color cue, leading to facilitating the predicted target color (the predicted color cue) and suppressing the predicted distractor color (the non-predicted color cue). In epoch 4 , the main effects of cue color and validity and the interaction were not significant, $F(1,43)=.02, p=$ $.89, \eta_{p}^{2}<.01, F(1,43)=.31, p=.58, \eta_{p}^{2}<.01$, and $F(1,43)=.23, p=.63, \eta_{p}^{2}<.01$. The cue validity effect (invalid RTs - valid RTs) for each type of cue and each epoch is reported in Fig. 6 (see Table 5 for invalid and valid RTs).

New ANOVAs (see Appendix Table 9) on cue color and cue validity were conducted for RT averaged across every 80 trials before the mismatch trials $(1-80,81-160,161-$ 240, 241-320, 321-400, 401-480, 481-560, 561-640, 641720 , and 721-800 trials; see Fig. 6). No significant interactions between cue color and cue validity (no nonspatial context-driven search effect) were found in any of the epochs (all $p s>.08$ ).

Accuracy No interactions between color and validity were significant in any of the epochs. In epoch 1 , the main effects of cue color and validity were not significant, $F(1,43)=1.65, p=.20$, $\eta_{p}^{2}=.03$, and $F(1,43)=5.53, p=.02, \eta_{p}^{2}=.11$, respectively. The interaction between cue color and validity was also not significant, $F(1,43)=.25, p=.61, \eta_{p}^{2}<.01$. In epoch 2 , the main effects of cue color and validity were not significant, $F(1,43)=$ $2.0, p=.16, \eta_{p}^{2}=.04$, and $F(1,43)=.63, p=.43, \eta_{p}^{2}=.01$, respectively. The interaction between cue color and validity was also not significant, $F(1,43)=1.09, p=.30, \eta_{p}^{2}=.03$. In epoch 3 , the main effect of cue color was not significant and the main effect of validity significant, $F(1,43)=2.86, p=.09, \eta_{p}^{2}=$ .06 , and $F(1,43)=8.76, p=.005, \eta_{p}^{2}=.16$, respectively. Critically, no interaction was observed, $F(1,43)<.01, p=$ $.93, \eta_{p}^{2}<.001$. Additionally, Bayes factor $\left(\mathrm{BF}_{01}\right)=4.5$ indicated that the null was 4.5 times more likely than the interaction, supporting no evidence of speed-accuracy trade-off. In epoch 4 , the main effects of cue color and validity were not significant, $F(1,43)=.19, p=.66, \eta_{p}^{2}<.01$, and $F(1,43)=.31, p=.58, \eta_{p}^{2}<$ .01 , respectively. The interaction between cue color and validity was also not significant, $F(1,43)<.01, p=.93, \eta_{p}^{2}<.001$ (see Table 6).

Awareness question No participant reported noticing the association between the context and color of the target.

\section{Discussion}

The current experiment removed the break and replicated the results of Experiments 1 and 2: the non-spatial context-driven search was found only in epoch 3 . Accordingly, the finding of Experiment 3 suggests that the non-spatial context-driven search was due to the mismatch trials rather than the break.

\section{General discussion}

The primary finding of the current study, replicated across three experiments, is that shape contexts can trigger attentional control settings for a target color predicted by the contexts (non-spatial context-driven search). Despite extensive evidence showing attentional control settings for non-spatial features such as color and shape in visual search (see Lamy \& Kristjánsson, 2013; Maunsell \& Treue, 2006, for reviews), the amount of evidence for non-spatial context-driven search (Anderson, 2015; Gozli et al., 2014) is low compared to evidence for spatial context-driven search (Brockmole et al., 2006; Chun, 2000; Chun \& Jiang, 1998, 2003; Jiang \& Wagner, 2004; Jiang et al., 2013; Olson \& Chun, 2002; Rosenbaum \& Jiang, 2013). This study adds to evidence for non-spatial context-driven search and demonstrates conditions under which non-spatial context-driven search may or may not be evident. Specifically, the non-spatial context-driven search occurred only after a transient violation in a learned association between a shape context and a target color. This suggests that context-driven search requires sufficient cognitive resources for expression of contexts guiding attention (Jiang \& Leung, 2005; Manginelli et al., 2013).

The lack of a non-spatial context-driven search effect prior to the mismatch trials and the presence of an effect after the mismatch trials suggests that the expression of the contextdriven cueing effect will be absent if cognitive resources are not sufficiently allocated to the learned association. Two possible mechanisms by which the mismatch trials can increase cognitive resources are exploratory attention and conflictdriven cognitive control. In associative learning, when previously learned associations are violated, exploratory attention increases attentional resources to the uncertain stimuli to be resolved (Easdale et al., 2019; Griffiths et al., 2011; Hall \& Pearce, 1982; Wills et al., 2007; Wilson et al., 1992). Furthermore, detection of cognitive conflict triggers an upregulation in cognitive control by allocating cognitive resources to the task-relevant information (Egner \& Hirsch, 2005). The shape context predicted the color of the targets. Therefore, an up-regulation in cognitive control would lead to an increase in cognitive resources for the shape/color associations. Although the shape contexts could not trigger attentional control settings for the shape-predicted color before the mismatch trials due to insufficient cognitive resources for the 


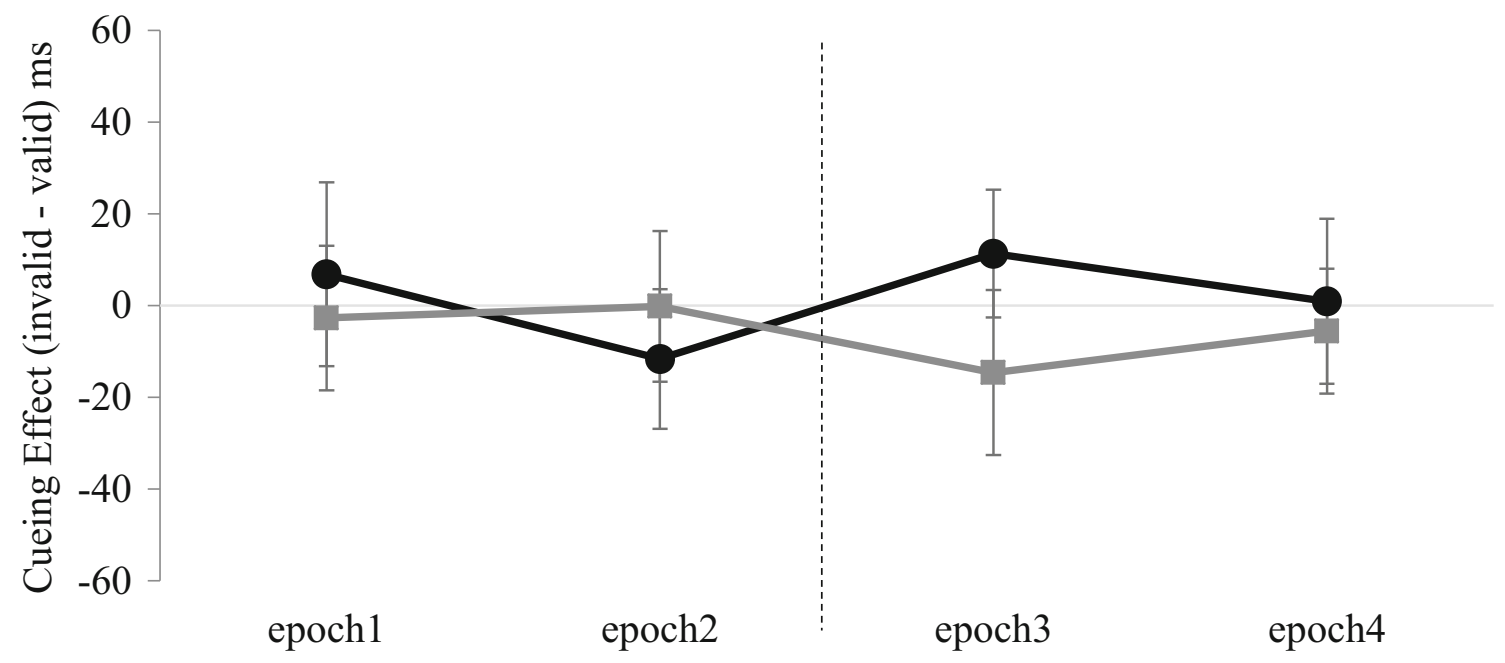

$\rightarrow-$ Predicted Color Cue $\rightarrow-$ Non-Predicted Color Cue
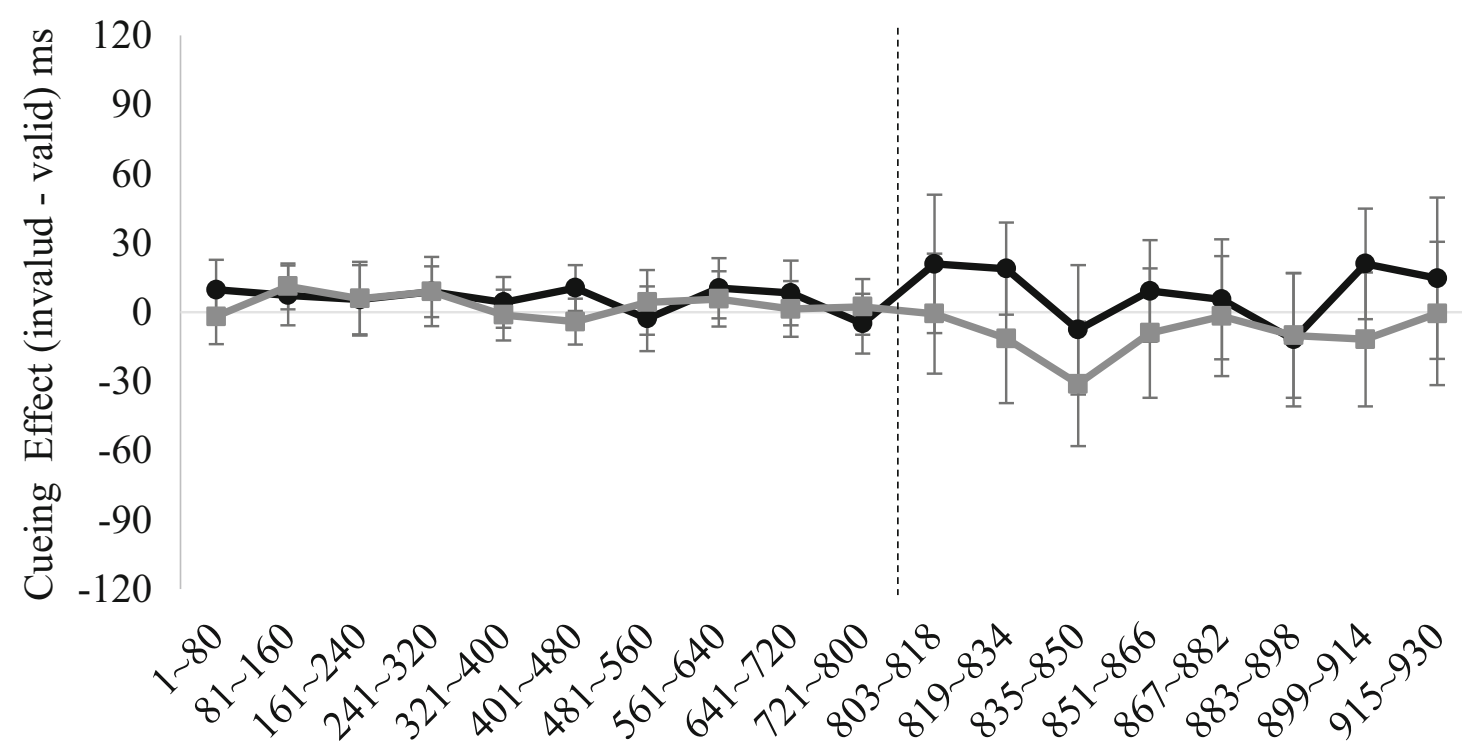

\section{Trials}

Fig. 6 Results of Experiment 3. In the top graph, each of epochs 1-4 includes 48 trials and across the following range of trials: 705-752, 753$800,803-850$, and $851-898$, respectively. The bottom graph is the data in bins of 80 trials until the mismatch trials and then bins of 16 trials after the

Table 5 Mean response times and standard error of the mean (in milliseconds) as a function of epoch, cue color, and cue validity in Experiment 3

\begin{tabular}{llllll}
\hline & \multicolumn{2}{l}{ Predicted color } & & \multicolumn{2}{l}{ Non-predicted color } \\
\cline { 2 - 3 } \cline { 6 - 6 } & Valid & Invalid & & Valid & Invalid \\
\hline Epoch 1 & $583(8)$ & $590(10)$ & & $590(9)$ & $587(10)$ \\
Epoch 2 & $586(9)$ & $575(8)$ & & $580(6)$ & $580(9)$ \\
Epoch 3 & $553(7)$ & $565(7)$ & & $569(6)$ & $555(8)$ \\
Epoch 4 & $567(9)$ & $568(7)$ & & $569(7)$ & $564(9)$ \\
\hline
\end{tabular}

mismatch trials. The mismatch trials occurred between epochs 2 and 3, on trials 801 and 802 (see dashed black vertical line). Error bars represent $95 \%$ confidence intervals

Table 6 Mean of accuracy and standard error of the mean (in percentage) as a function of epoch, cue color, and cue validity in Experiment 3

\begin{tabular}{llllll}
\hline & \multicolumn{2}{l}{ Predicted color } & & \multicolumn{2}{l}{ Non-predicted color } \\
\cline { 2 - 3 } \cline { 6 - 6 } \cline { 6 - 6 } & Valid & Invalid & & Valid & Invalid \\
\hline Epoch 1 & $89.2(1.8)$ & $92.8(1.3)$ & & $91.3(1.5)$ & $93.9(1.2)$ \\
Epoch 2 & $91.7(1.5)$ & $91.9(1.2)$ & & $94.1(1.0)$ & $92.2(1.5)$ \\
Epoch 3 & $93.6(1.0)$ & $96.4(.09)$ & & $91.9(1.5)$ & $94.9(1.0)$ \\
Epoch 4 & $93.4(1.1)$ & $92.6(1.4)$ & & $93.8(1.3)$ & $93.2(1.3)$ \\
\hline
\end{tabular}


shape/color association, the mismatch trials allow the necessary cognitive resources for shape contexts to trigger attentional control for the associated color.

The non-spatial context-driven search by the aid of the mismatch trials increasing cognitive resources for the shape/color association is consistent with previous findings. In previous studies showing non-spatial context-driven attentional sets, a methodological factor existed that increased allocation of cognitive resources to the task. Anderson (2015) found the shape contexts triggering attentional control setting for color when the shape/color association involved reward learning. Reward is a critical factor enhancing associative learning (Hyman et al., 2006). Gozli et al. (2014) found the non-spatial context-driven search when a single object contained both the shape and color features so that the shape/color association received attentional benefits. However, they did not find the non-spatial contextdriven search without the conjunctive presentation because such the attentional benefits to the shape/color association are removed. In line with this, in the current study, without the aid of the mismatch trials, the non-spatial context-driven search was not found (prior to the mismatch trials). Therefore, the use of learned shape/color associations to guide attention appears to require a boost in cognitive resources.

Although the spatial context-driven search was commonly found without methodological factors increasing cognitive resources for the association of spatial features (Brockmole et al., 2006; Chun, 2000; Chun \& Jiang, 1998, 2003; Jiang \& Wagner, 2004; Jiang, Sigstad, \& Swallow, 2013; Olson \& Chun, 2002; Rosenbaum \& Jiang, 2013; see Goujon et al., 2015 for review), it was interrupted when there were obstructers preventing the spatial association from receiving cognitive resources (Jiang \& Leung, 2005; Manginelli et al., 2013). The spatial context-driven search did not occur when contexts received few attentional resources (Jiang \& Leung, 2005) or when working memory was used for a different task (Manginelli et al., 2013). Taken together with the non-spatial context-driven search requiring additional cognitive resources, the spatial context-driven search not requiring the additional cognitive resources but being interrupted by the obstructers suggests that cognitive resources modulate the context-driven search and supports that the context-driven search requires more cognitive resources for the non-spatial than spatial context-driven search. A different allocation of cognitive resources required for the non-spatial and spatial context-driven search might in part account for rare evidence of the non-spatial compared to spatial context-driven search despite the ability to establish attentional control settings for non-spatial features such as color and shape (Lamy \& Kristjánsson, 2013; Maunsell \& Treue, 2006).

The requirement of more cognitive resources for the nonspatial than spatial associative learning is also consistent with the finding that memory for spatial information is used more than non-spatial information of distracters to guide attention during search (Beck, Peterson, \& Vomela, 2006). For example, when locations of the previously attended distracters were changed, search was interrupted. However, when identity (color and shape) of attended distractors was changed, search was not interrupted, suggesting that spatial rather than nonspatial information was used to guide attention. In other words, to use non-spatial information during search, additional task situations by which more cognitive resources are allocated to the non-spatial information may be needed. In the non-spatial context-driven search, the situations were made via methodological factors that boost available cognitive resources.

The non-spatial context-driven search in the present study appears to be the result of the trend of both the validity effect of the predicted color cue and the reversed validity effect of the non-predicted color cue. This pattern is not surprising given the fact that the contexts predicted both target color (the same color as the predicted color cue) and distractor color (the same color as the non-predicted color cue). Known target-features can be facilitated, increasing attention to the targets (Desimone \& Duncan, 1995; Wolfe, 1994). In addition, known non-target-features can be suppressed, preventing attention to the non-targets (Arita, Carlisle, \& Woodman, 2012; Geng, DiQuattro, \& Helm, 2017). These general attentional abilities of the facilitation (for selection) and suppression (for filtering out) were found in a previous search study (Ogawa, Takeda, \& Kumada, 2007) where the contexts triggered the attentional facilitation of the context-predicted target information and attentional suppression of the context-predicted distractor information. In the pre-cueing paradigm, the attentional facilitation and suppression are indicated by the validity and reversed validity effects, respectively (e.g., Belopolsky, Schreij, $\&$ Theeuwes, 2010; Kerzel, 2019). Thus, the trend of the validity effect of the predicted color cue and the reversed validity effect of the non-predicted color cue in the present study would reflect the contexts predicting target color and distractor color.

The use of the shape/color associations immediately after the mismatch trials implies that the associations were already learned before the mismatch trials. That is, the associative learning occurred automatically, but the use of this learned information did not. This is in line with previous research (Jiang \& Leung, 2005; Manginelli et al., 2013) where cognitive resources (working memory in Manginelli et al., 2013, attention in Jiang \& Leung, 2005) were needed for the use (expression) of the learned associations to guide attention, whereas learning the associations did not depend on cognitive resources.

In a close inspection of the first 16 trials after the mismatch trials, the non-spatial context-driven search appears to be slightly delayed in Experiment 1 compared to Experiments 2 and 3. One possibility is that this is due simply to noise in the 
data given that there are only 16 trials in each epoch. Another possibility is that this difference is due to the use of different types of mismatch trials. The mismatch trials in Experiment 1 were the reverse of the match trials, whereas in Experiments 2 and 3 color was removed in the mismatch trials. Therefore, in Experiment 1, a reversed non-spatial context-driven search effect (a non-predicted color cue capturing attention more than a predicted color cue) might be slightly induced by the two mismatch trials having the opposite color-shape associations. This might have led to the slight delay in Experiment 1 compared to Experiments 2 and 3.
In conclusion, the present study demonstrated that associations between shape and color can be learned, and then the shape contexts can guide attention to the shape-predicted color. However, this non-spatial context-driven effect may fail to be used to guide attention without a boost in cognitive resources (Gozli et al., 2014) that does not appear to be necessary for spatial context-driven search (Chun \& Jiang, 1998). This is also in line with research other than context-driven search showing that spatial information is processed more automatically during search than non-spatial information search (Beck et al., 2006).

\section{Appendix 1}

Table 7 Experiment 1 ANOVAs for bins of 80 trials across experimental trials prior to the mismatch trials.

\begin{tabular}{|c|c|c|c|c|c|c|c|c|c|c|c|}
\hline & Trials & $1-80$ & $\begin{array}{l}81- \\
160\end{array}$ & $\begin{array}{l}161- \\
240\end{array}$ & $\begin{array}{l}241- \\
320\end{array}$ & $\begin{array}{l}321- \\
400\end{array}$ & $\begin{array}{l}401- \\
480\end{array}$ & $\begin{array}{l}481- \\
560\end{array}$ & $\begin{array}{l}561- \\
640\end{array}$ & $\begin{array}{l}641- \\
720\end{array}$ & $\begin{array}{l}721- \\
800\end{array}$ \\
\hline \multirow[t]{3}{*}{ Color } & $F$ & 0.032 & 1.4 & 0.4 & 1.479 & 2.368 & 2.721 & 0.019 & 0.079 & 0.179 & 3.326 \\
\hline & $P$ & 0.859 & 0.249 & 0.533 & 0.236 & 0.137 & 0.113 & 0.891 & 0.781 & 0.676 & 0.081 \\
\hline & $\eta_{p}^{2}$ & 0.001 & 0.057 & 0.017 & 0.06 & 0.093 & 0.106 & 0.001 & 0.003 & 0.008 & 0.126 \\
\hline \multirow[t]{3}{*}{ Validity } & $F$ & 3.743 & 0.223 & 1.378 & 1.878 & 1.478 & 0.836 & 1.458 & 4.39 & 0.02 & 3.183 \\
\hline & $P$ & 0.065 & 0.641 & 0.252 & 0.184 & 0.236 & 0.37 & 0.239 & 0.047 & 0.888 & 0.088 \\
\hline & $\eta_{p}^{2}$ & 0.14 & 0.01 & 0.057 & 0.075 & 0.06 & 0.035 & 0.06 & 0.16 & 0.001 & 0.122 \\
\hline \multirow[t]{3}{*}{ Interaction } & $F$ & 0.188 & 1.81 & 0.624 & 0.552 & 0.309 & 0.304 & 0.001 & 0.122 & 0.202 & 0.639 \\
\hline & $P$ & 0.669 & 0.192 & 0.437 & 0.465 & 0.583 & 0.587 & 0.98 & 0.73 & 0.658 & 0.432 \\
\hline & $\eta_{p}^{2}$ & 0.008 & 0.073 & 0.026 & 0.023 & 0.013 & 0.013 & $<0.001$ & 0.005 & 0.009 & 0.027 \\
\hline
\end{tabular}

\section{Appendix 2}

Table 8 Experiment 2 ANOVAs for bins of 80 trials across experimental trials prior to the mismatch trials.

\begin{tabular}{|c|c|c|c|c|c|c|c|c|c|c|c|}
\hline & Trials & $1-80$ & $81-160$ & $\begin{array}{l}161- \\
240\end{array}$ & $\begin{array}{l}241- \\
320\end{array}$ & $\begin{array}{l}321- \\
400\end{array}$ & $\begin{array}{l}401- \\
480\end{array}$ & $\begin{array}{l}481- \\
560\end{array}$ & $\begin{array}{l}561- \\
640\end{array}$ & $\begin{array}{l}641- \\
720\end{array}$ & $\begin{array}{l}721- \\
800\end{array}$ \\
\hline \multirow[t]{3}{*}{ Color } & $F$ & 0.448 & 1.496 & 0.364 & 0.495 & 0.276 & 0.306 & 0.027 & 1.827 & 0.302 & $<0.001$ \\
\hline & $P$ & 0.51 & 0.234 & 0.552 & 0.489 & 0.605 & 0.585 & 0.87 & 0.19 & 0.588 & 0.992 \\
\hline & $\eta_{p}^{2}$ & 0.019 & 0.061 & 0.016 & 0.021 & 0.012 & 0.013 & 0.001 & 0.074 & 0.013 & $<0.001$ \\
\hline \multirow[t]{3}{*}{ Validity } & $F$ & 0.411 & 0.011 & 0.536 & 0.1 & 0.007 & 0.017 & 0.871 & 2.156 & 0.279 & 5.728 \\
\hline & $p$ & 0.528 & 0.916 & 0.471 & 0.755 & 0.935 & 0.898 & 0.36 & 0.156 & 0.603 & 0.025 \\
\hline & $\eta_{p}^{2}$ & 0.018 & $<0.001$ & 0.023 & 0.004 & $<0.001$ & 0.001 & 0.036 & 0.086 & 0.012 & 0.199 \\
\hline \multirow[t]{3}{*}{ Interaction } & $F$ & 0.124 & 0.598 & 0.71 & 0.028 & 3.794 & 0.947 & 6.034 & 0.629 & 0.384 & 0.033 \\
\hline & $p$ & 0.728 & 0.447 & 0.408 & 0.869 & 0.064 & 0.341 & 0.022 & 0.436 & 0.542 & 0.857 \\
\hline & $\eta_{p}^{2}$ & 0.005 & 0.025 & 0.03 & 0.001 & 0.142 & 0.04 & 0.208 & 0.027 & 0.016 & 0.001 \\
\hline
\end{tabular}




\section{Appendix 3}

Table 9 Experiment 3 ANOVAs for bins of 80 trials across experimental trials prior to the mismatch trials.

\begin{tabular}{|c|c|c|c|c|c|c|c|c|c|c|c|}
\hline & Trials & $1-80$ & $\begin{array}{l}81- \\
160\end{array}$ & $\begin{array}{l}161- \\
240\end{array}$ & $\begin{array}{l}241- \\
320\end{array}$ & $\begin{array}{l}321- \\
400\end{array}$ & $\begin{array}{l}401- \\
480\end{array}$ & $\begin{array}{l}481- \\
560\end{array}$ & $\begin{array}{l}561- \\
640\end{array}$ & $\begin{array}{l}641- \\
720\end{array}$ & $\begin{array}{l}721- \\
800\end{array}$ \\
\hline \multirow[t]{3}{*}{ Color } & $F$ & 5.563 & 0.459 & 3.427 & 1.590 & 0.039 & 0.811 & 0.294 & 0.444 & 1.107 & 0.505 \\
\hline & $p$ & 0.023 & 0.502 & 0.071 & 0.214 & 0.844 & 0.373 & 0.590 & 0.509 & 0.299 & 0.481 \\
\hline & $\eta_{p}^{2}$ & 0.115 & 0.011 & 0.074 & 0.036 & 0.001 & 0.019 & 0.007 & 0.010 & 0.025 & 0.012 \\
\hline \multirow[t]{3}{*}{ Validity } & $F$ & 0.732 & 3.976 & 1.014 & 3.068 & 0.139 & 0.932 & 0.021 & 3.800 & 1.298 & 0.102 \\
\hline & $p$ & 0.397 & 0.053 & 0.320 & 0.087 & 0.711 & 0.340 & 0.886 & 0.058 & 0.261 & 0.751 \\
\hline & $\eta_{p}^{2}$ & 0.017 & 0.085 & 0.023 & 0.067 & 0.003 & 0.021 & $<0.001$ & 0.081 & 0.029 & 0.002 \\
\hline \multirow[t]{3}{*}{ Interaction } & $F$ & 1.610 & 0.241 & 0.002 & $<0.001$ & 0.480 & 3.150 & 0.550 & 0.245 & 0.422 & 0.562 \\
\hline & $p$ & 0.211 & 0.626 & 0.965 & 0.999 & 0.492 & 0.083 & 0.462 & 0.623 & 0.519 & 0.457 \\
\hline & $\eta_{p}^{2}$ & 0.036 & 0.006 & $<0.001$ & $<0.001$ & 0.011 & 0.068 & 0.013 & 0.006 & 0.010 & 0.013 \\
\hline
\end{tabular}

\section{References}

Anderson, B. A. (2015). Value-driven attentional priority is context specific. Psychonomic Bulletin \& Review, 22(3), 750-756.

Arita, J. T., Carlisle, N. B., \& Woodman, G. F. (2012). Templates for rejection: configuring attention to ignore task-irrelevant features. Journal of experimental psychology: human perception and performance, 38(3), 580-584.

Awh, E., Belopolsky, A. V., \& Theeuwes, J. (2012). Top-down versus bottom-up attentional control: a failed theoretical dichotomy. Trends in Cognitive Science, 16(8), 437-443.

Baker, C. I., Olson, C. R., \& Behrmann, M. (2004). Role of attention and perceptual grouping in visual statistical learning. Psychological Science, 15(7), 460-466.

Beck, M. R., Peterson, M. S., \& Vomela, M. (2006). Memory for where, but not what, is used during visual search. Journal of Experimental Psychology: Human Perception and Performance, 32(2), 235-250.

Beck, M. R., Angelone, B. L., Levin, D. T., Peterson, M. S., \& Varakin, D. A. (2008). Implicit learning for probable changes in a visual change detection task. Consciousness and cognition, 17(4), 11921208 .

Beck, M. R., Goldstein, R. R., van Lamsweerde, A. E., \& Ericson, J. M. (2018). Attending globally or locally: Incidental learning of optimal visual attention allocation. Journal of experimental psychology: learning, memory, and cognition, 44(3), 387-398.

Belopolsky, A. V., Schreij, D., \& Theeuwes, J. (2010). What is top-down about contingent capture? Attention, Perception, \& Psychophysics, 72(2), 326-341

Botvinick, M. M., Cohen, J. D., \& Carter, C. S. (2004). Conflict monitoring and anterior cingulate cortex: an update. Trends in cognitive sciences, 8(12), 539-546.

Brockmole, J. R., Castelhano, M. S., \& Henderson, J. M. (2006). Contextual cueing in naturalistic scenes: Global and local contexts. Journal of Experimental Psychology: Learning, Memory, and Cognition, 32(4), 699-706.

Chun, M. M. (2000). Contextual cuing of visual attention. Trends in Cognitive Science, 4(5), 170-178.
Chun, M. M., \& Jiang, Y. (1998). Contextual cuing: Implicit learning and memory of visual context guides spatial attention. Cognitive Psychology, 36, 28-71.

Chun, M. M., \& Jiang, Y. (1999). Top-down attentional guidance based on implicit learning of visual covariation. Psychological Science, 10, 360- 365 .

Chun, M. M., \& Jiang, Y. (2003). Implicit, long-term spatial contextual memory. Journal of Experimental Psychology: Learning, Memory, and Cognition, 29(2), 224-234.

Cosman, J. D., \& Vecera, S. P. (2014). Establishment of an attentional set via statistical learning. Journal of Experimental Psychology: Human Perception and Performance, 40, 1-6.

Den Ouden, H. E., Friston, K. J., Daw, N. D., McIntosh, A. R., \& Stephan, K. E. (2008). A dual role for prediction error in associative learning. Cerebral cortex, 19(5), 1175-1185.

Desimone, R., \& Duncan, J. (1995). Neural mechanisms of selective visual attention. Annual review of neuroscience, 18(1), 193-222.

Endo, N., \& Takeda, Y. (2004). Selective learning of spatial configuration and object identity in visual search. Perception \& Psychophysics, 66(2), 293-302.

Easdale, L. C., Le Pelley, M. E., \& Beesley, T. (2019). The onset of uncertainty facilitates the learning of new associations by increasing attention to cues. The Quarterly Journal of Experimental Psychology, 72, 193-208.

Egner, T. (2007). Congruency sequence effects and cognitive control. Cognitive, Affective, \& Behavioral Neuroscience, 7(4), 380-390.

Egner, T., \& Hirsch, J. (2005). Cognitive control mechanisms resolve conflict through cortical amplification of task-relevant information. Nature neuroscience, 8(12), 1784-1790.

Fiser, J., \& Aslin, R. N. (2005). Encoding multielement scenes: Statistical learning of visual feature hierarchies. Journal of Experimental Psychology: General, 134, 521-537.

Folk, C. L., \& Remington, R. W. (1998). Selectivity in distraction by irrelevant featural singletons: Evidence for two forms of attentional capture. Journal of Experimental Psychology: Human Perception and Performance, 24, 847-858.

Geng, J. J., DiQuattro, N. E., \& Helm, J. (2017). Distractor probability changes the shape of the attentional template. Journal of 
Experimental Psychology: Human Perception and Performance, 43, 1993-2007.

Goujon, A., Didierjean, A., \& Thorpe, S. (2015). Investigating implicit statistical learning mechanisms through contextual cueing. Trends in cognitive sciences, 19(9), 524-533.

Gozli, D. G., Moskowitz, J. B., \& Pratt, J. (2014). Visual attention to features by associative learning. Cognition, 133(2), 488-501.

Griffiths, O., Johnson, A. M., \& Mitchell, C. J. (2011). Negative transfer in human associative learning. Psychological science, 22(9), 11981204.

Hall, G., \& Pearce, J. M. (1982). Restoring the associability of a preexposed CS by a surprising event. The Quarterly Journal of Experimental Psychology Section B, 34(3b), 127-140.

Hyman, S. E., Malenka, R. C., \& Nestler, E. J. (2006). Neural mechanisms of addiction: the role of reward-related learning and memory. Annu. Rev. Neurosci., 29, 565-598.

Jiang, Y., \& Chun, M. M. (2001). Selective attention modulates implicit learning. The Quarterly Journal of Experimental Psychology, 54A, $1105-1124$.

Jiang, Y., \& Leung, A. W. (2005). Implicit learning of ignored visual context. Psychonomic Bulletin \& Review, 12(1), 100-106.

Jiang, Y. V., Sigstad, H. M., \& Swallow, K. M. (2013). The time course of attentional deployment in contextual cueing. Psychonomic Bulletin \& Review, 20(2), 282-288.

Jiang, Y., \& Wagner, L. C. (2004). What is learned in spatial contextual cuing - configuration or individual locations? Perception \& Psychophysics, 66(3), 454-463.

Kahneman, D., Treisman, A., \& Gibbs, B. J. (1992). The reviewing of object files: Object-specific integration of information. Cognitive psychology, 24(2), 175-219.

Kaye, H., \& Pearce, J. M. (1984). The strength of the orienting response during Pavlovian conditioning. Journal of Experimental Psychology: Animal Behavior Processes, 10(1), 90-109.

Kerzel, D. (2019). The precision of attentional selection is far worse than the precision of the underlying memory representation. Cognition, 186, 20-31.

Kirkham, N. Z., Slemmer, J. A., \& Johnson, S. P. (2002). Visual statistical learning in infancy: Evidence for a domain general learning mechanism. Cognition, 83(2), B35-B42.

Lamy, D. F., \& Kristjánsson, A. (2013). Is goal-directed attentional guidance just intertrial priming? A review. Journal of Vision, 13, 1-19.

Leber, A. B., Gwinn, R. E., Hong, Y., \& O’Toole, R. J. (2016). Implicitly learned suppression of irrelevant spatial locations. Psychonomic Bulletin \& Review, 23(6), 1873-1881.

Manginelli, A. A., Langer, N., Klose, D., \& Pollmann, S. (2013). Contextual cueing under working memory load: Selective interference of visuospatial load with expression of learning. Attention, Perception, \& Psychophysics, 75(6), 1103-1117.

Maunsell, J. H., \& Treue, S. (2006). Feature-based attention in visual cortex. Trends in neurosciences, 29(6), 317-322.

Mayr, U., Awh, E., \& Laurey, P. (2003). Conflict adaptation effects in the absence of executive control. Nature neuroscience, 6(5), 450-452.

Ogawa, H., Takeda, Y., \& Kumada, T. (2007). Probing attentional modulation of contextual cueing. Visual Cognition, 15(3), 276-289.
Olson, I. R., \& Chun, M. M. (2002). Perceptual constraints on implicit learning of spatial context. Visual cognition, 9(3), 273-302.

Otsuka, S., Nishiyama, M., Nakahara, F., \& Kawaguchi, J. (2013). Visual statistical learning based on the perceptual and semantic information of objects. Journal of Experimental Psychology: Learning, Memory, and Cognition, 39(1), 196-207.

Pearce, J. M., \& Bouton, M. E. (2001). Theories of associative learning in animals. Annual review of psychology, 52(1), 111-139.

Pearce, J. M., \& Hall, G. (1980). A model for Pavlovian learning: variations in the effectiveness of conditioned but not of unconditioned stimuli. Psychological review, 87(6), 532-552.

Rey-Mermet, A., \& Meier, B. (2017). How long-lasting is the postconflict slowing after incongruent trials? Evidence from the Stroop, Simon, and flanker tasks. Attention, Perception, \& Psychophysics, 79(7), 1945-1967.

Rosenbaum, G. M., \& Jiang, Y. V. (2013). Interaction between scenebased and array-based contextual cueing. Attention, Perception, \& Psychophysics, 75(5), 888-899.

Swan, J. A., \& Pearce, J. M. (1988). The orienting response as an index of stimulus associability in rats. Journal of Experimental Psychology: Animal Behavior Processes, 14(3), 292-301.

Torralba, A., Oliva, A., Castelhano, M. S., \& Henderson, J. M. (2006). Contextual guidance of eye movements and attention in real-world scenes: the role of global features in object search. Psychological review, 113(4), 766-786.

Turk-Browne, N. B., Jungé, J. A., \& Scholl, B. J. (2005). The automaticity of visual statistical learning. Journal of Experimental Psychology: General, 134(4), 552-564.

Vaidya, C. J., Huger, M., Howard, D. V., \& Howard Jr, J. H. (2007). Developmental differences in implicit learning of spatial context. Neuropsychology, 21(4), 497-506.

Wills, A. J., Lavric, A., Croft, G. S., \& Hodgson, T. L. (2007). Predictive learning, prediction errors, and attention: Evidence from eventrelated potentials and eye tracking. Journal of Cognitive Neuroscience, 19(5), 843-854.

Wilson, P. N., Boumphrey, P., \& Pearce, J. M. (1992). Restoration of the orienting response to a light by a change in its predictive accuracy. The Quarterly Journal of Experimental Psychology Section B, 44(1b), 17-36.

Wolfe, J. M. (1994). Guided search 2.0: A revised model of visual search. Psychonomic Bulletin \& Review, 1, 202-238.

Open Practices Statement The experiments were not preregistered.

The data from both experiments are available at the Center for Open Science: https://osf.io/ejdwm/.

Publisher's note Springer Nature remains neutral with regard to jurisdictional claims in published maps and institutional affiliations. 